\title{
THE WHITEHEAD FIRST LEMMA FOR ALTERNATIVE ALGEBRAS ${ }^{1}$
}

EARL JAY TAFT

1. Introduction. The Whitehead first lemma for associative algebras states that any derivation of a separable algebra into a bimodule is inner. In [6], Schafer has proved the analogue of this result for alternative algebras over a field of characteristic zero. He does this by using the corresponding result of Jacobson, [3], for the Jordan algebra and Jordan module formed from an alternative algebra and bimodule. Recent structure theory, due to Jacobson [4] indicates that it may be more natural to first prove this kind of result for alternative algebras, and then use this to prove the analogous result for Jordan algebras. In this paper, we prove the Whitehead first lemma for alternative algebras using only the alternative structure theory. Moreover, the result holds for any base field of characteristic not two or three.

The proof, as given in $\$ 3$, is a reduction to some special cases. Hence we start with a discussion of derivations in Cayley algebras. The numbers in square brackets refer to the bibliography at the end of the text. All algebras in this paper are finite-dimensional over the base field.

2. Derivations in a Cayley algebra. It is assumed that the characteristic of the base field $\Phi$ is not two or three. If $\mathfrak{A}$ is a (nonassociative) algebra over $\Phi$, let $\mathfrak{D}(\mathfrak{A})$ denote the Lie algebra of derivations of $\mathfrak{A}$ over $\Phi$. A derivation is inner if it is contained in the enveloping Lie algebra of the right and left multiplications by elements of $\mathfrak{A}$ (cf. [5]).

If $\mathfrak{Q}$ is a generalized quaternion algebra over $\Phi$, then the generalized Cayley algebra over $\Phi$ is $\mathfrak{S}=\mathfrak{Q} 1+\mathfrak{Q} e_{4}$, with a multiplication given by $\left(a+b e_{4}\right)\left(c+d e_{4}\right)=\left(a c+\bar{d} b \alpha_{4}\right)+(d a+b \bar{c}) e_{4}$, where $\alpha_{4} \in \Phi$, $\alpha_{4} \neq 0, a, b, c, d \in \mathfrak{Q}$. If $\mathfrak{Q}$ has a basis $\left(1, e_{1}, e_{2}, e_{3}\right)$, then set $e_{5}=e_{1} e_{4}$, $e_{6}=e_{4} e_{2}$, and $e_{7}=e_{3} e_{4}$. Then $1, e_{1}, e_{2}, \cdots, e_{7}$ is a basis for $\mathfrak{E}$. If $q \rightarrow \vec{q}$ denotes the involution in $\mathfrak{Q}$, then $\mathfrak{S}$ has the involution $x=q_{1}+q_{2} e_{4} \rightarrow \bar{x}$ $=\bar{q}_{1}-q_{2} e_{4}$. $\mathbb{E}$ is an alternative algebra, and $\mathfrak{S}=\Phi 1 \oplus \mathbb{S}^{1}$, where $\mathfrak{\mho}^{1}$ is the space of elements of trace zero. (Trace $x=x+\bar{x}$ ). Since $e_{1}, e_{2}, e_{4}$ generate $\mathbb{E}$, any derivation is uniquely determined by its action on $e_{1}, e_{2}, e_{4}$. Jacobson has shown, [2], that $\mathfrak{S}^{1}$ is an invariant $\mathfrak{D}(\mathfrak{C})$ -

Received by the editors March 14, 1957.

1 The material in this paper is a portion of a dissertation submitted to Yale University, prepared under the guidance of Professor Nathan Jacobson. 
subspace, and that if $e_{1} D=\sum_{i=1}^{7} \lambda_{i} e_{i}, e_{2} D=\sum_{i=1}^{7} \mu_{i} e_{i}, e_{4} D=\sum_{i=1}^{7} \gamma_{i} e_{i}$, then

$$
\begin{aligned}
\lambda_{1} & =\mu_{2}=\nu_{4}=\mu_{1} \alpha_{1}+\lambda_{2} \alpha_{2}=\nu_{1} \alpha_{1}+\lambda_{4} \alpha_{4}=\nu_{2} \alpha_{2}+\mu_{4} \alpha_{4} \\
& =\lambda_{6} \alpha_{4} \alpha_{2}+\mu_{5} \alpha_{1} \alpha_{4}-\nu_{3} \alpha_{1} \alpha_{2}=0 .
\end{aligned}
$$

(Note: $\alpha_{1}=e_{1}^{2}, \alpha_{2}=e_{2}^{2}$ ). It follows that $\mathfrak{D}(\mathfrak{S})$ is a fourteen-dimensional Lie algebra. Using (1), we display a basis for $\mathfrak{D}(\mathfrak{E})$, denoted $D_{1}$, $D_{2}, \cdots, D_{14}$. Only the effect on $e_{1}, e_{2}, e_{4}$ is shown.

\begin{tabular}{c|c|c|c} 
& $e_{1}$ & $e_{2}$ & $e_{4}$ \\
\hline$D_{1}$ & $e_{2}$ & $-\alpha_{2} \alpha_{1}^{-1} e_{1}$ & 0 \\
\hline$D_{2}$ & $e_{3}$ & 0 & $-\alpha_{4} \alpha_{1}^{-1} e_{1}$ \\
\hline$D_{3}$ & $e_{4}$ & 0 & 0 \\
\hline$D_{4}$ & $e_{5}$ & 0 & $\alpha_{4} \alpha_{1}^{-1} e_{3}$ \\
\hline$D_{5}$ & $e_{6}$ & 0 & 0 \\
\hline$D_{6}$ & $e_{7}$ & 0 & 0 \\
\hline$D_{7}$ & 0 & $e_{3}$ & $-\alpha_{4} \alpha_{2}^{-1} e_{2}$ \\
\hline$D_{8}$ & 0 & $e_{4}$ & $\alpha_{4} \alpha_{2}^{-1} e_{3}$ \\
\hline$D_{9}$ & 0 & $e_{5}$ & 0 \\
\hline$D_{10}$ & 0 & $e_{6}$ & $e_{5}$ \\
\hline$D_{11}$ & 0 & $e_{7}$ & $e_{7}$ \\
\hline$D_{12}$ & 0 & 0 & $e_{13}$ \\
\hline$D_{13}$ & 0 & 0 & 0 \\
\hline$D_{14}$ & 0 & 0 & $e_{5}$ \\
\hline
\end{tabular}

In any alternative algebra $\mathfrak{A}$, the linear transformation

$$
D_{x, y}=\left[R_{x}, R_{y}\right]+\left[R_{x}, L_{y}\right]+\left[L_{x}, L_{y}\right], \quad x, y \in \mathfrak{A}
$$

is a derivation, where $R_{x}, L_{x}$ denote right and left multiplication, respectively, by $x$ in $\mathfrak{A}$ (See [5]). Hence a linear transformation $\sum_{i} D_{x_{i}, y_{i}}$ is an inner derivation in $\mathfrak{A} . D_{x, y}$ is a bilinear function of $x$ and $y, D_{x, x}=0, D_{x, y}=-D_{y, x}$, and $D_{1, x}=D_{x, 1}=0$ for 1 an identity of $\mathfrak{A}$. Hence if $\left(1, e_{1}, \cdots, e_{n}\right)$ is a basis for $\mathfrak{A}$, then $\left\{\sum D_{x_{i}, y_{i}} ; x_{i}, y_{i} \in \mathfrak{A}\right\}$ 
has dimension at most $n(n-1) / 2$, and is spanned by $D_{e_{i}, e_{j}}$, for $i<j$.

Returning to $\sqrt{5}$, we now show that the space spanned by these twenty-one inner derivations is of dimension fourteen, and hence all derivations of $\mathbb{5}$ are inner and have the form $\sum D_{x_{i}, y_{i}}$. We choose fourteen $D_{e_{i}, e_{j}}$, tabulate their action of $e_{1}, e_{2}, e_{4}$, and then express them in terms of $D_{1}, D_{2}, \cdots, D_{14}$, noting their linear independence.

\begin{tabular}{|c|c|c|c|}
\hline & $e_{1}$ & $e_{2}$ & $e_{4}$ \\
\hline$D_{e_{1}, e_{2}}$ & $4 \alpha_{1} e_{2}$ & $-4 \alpha_{2} e_{1}$ & $2 e_{7}$ \\
\hline$D_{e_{4}, e_{7}}$ & $2 \alpha_{1} \alpha_{4} e_{2}$ & $-2 \alpha_{2} \alpha_{4} e_{1}$ & $4 \alpha_{4} e_{7}$ \\
\hline$D_{e_{1}, e_{3}}$ & $4 \alpha_{1} e_{3}$ & 0 & $-2 \alpha_{1} e_{6}$ \\
\hline$D_{e_{4}, e_{6}}$ & $-2 \alpha_{4} e_{3}$ & 0 & $4 \alpha_{4} e_{6}$ \\
\hline$D_{e_{1}, e_{4}}$ & $4 \alpha_{1} e_{4}$ & $-2 e_{7}$ & $-4 \alpha_{4} e_{1}$ \\
\hline$D_{e_{2}, e_{7}}$ & $-2 \alpha_{1} \alpha_{2} e_{4}$ & $4 \alpha_{2} e_{7}$ & $2 \alpha_{2} \alpha_{4} e_{1}$ \\
\hline$D_{e_{1}, e_{5}}$ & $4 \alpha_{1} e_{5}$ & $2 \alpha_{1} e_{6}$ & 0 \\
\hline$D_{e_{2}, e_{6}}$ & $2 \alpha_{2} e_{5}$ & $4 \alpha_{2} e_{6}$ & 0 \\
\hline$D_{e_{1}, e_{6}}$ & $4 \alpha_{1} e_{6}$ & $-2 \alpha_{2} e_{5}$ & $2 \alpha_{4} e_{3}$ \\
\hline$D_{e_{2}, e_{5}}$ & $-2 \alpha_{1} e_{6}$ & $4 \alpha_{2} e_{5}$ & $2 \alpha_{4} e_{3}$ \\
\hline$D_{e_{1}, e_{7}}$ & $4 \alpha_{1} e_{7}$ & $2 \alpha_{1} \alpha_{2} e_{4}$ & $-2 \alpha_{1} \alpha_{4} e_{2}$ \\
\hline$D_{e_{2}, e_{4}}$ & $2 e_{7}$ & $4 \alpha_{2} e_{4}$ & $-4 \alpha_{1} e_{2}$ \\
\hline$D_{e_{2}, e_{3}}$ & 0 & $4 \alpha_{2} e_{3}$ & $-2 \alpha_{2} e_{5}$ \\
\hline$D_{e_{4}, e_{5}}$ & 0 & $-2 \alpha_{4} e_{3}$ & $4 \alpha_{4} e_{5}$ \\
\hline
\end{tabular}

Also, the following equations hold.

$$
\begin{aligned}
& \left\{\begin{array}{l}
D_{e_{1}, e_{2}}=4 \alpha_{1} D_{1}+2 D_{14}, \\
D_{e_{4}, e_{7}}=2 \alpha_{1} \alpha_{4} D_{1}+4 \alpha_{4} D_{14},
\end{array}\right. \\
& \left\{\begin{array}{l}
D_{e_{1}, e_{3}}=4 \alpha_{1} D_{2}-2 \alpha_{1} D_{13}, \\
D_{e_{1}, e_{6}}=-2 \alpha_{4} D_{2}+4 \alpha_{4} D_{13},
\end{array}\right. \\
& \left\{\begin{array}{l}
D_{e_{1}, e_{4}}=4 \alpha_{1} D_{3}-2 D_{11}, \\
D_{e_{2}, e_{7}}=-2 \alpha_{1} \alpha_{2} D_{3}+4 \alpha_{2} D_{11},
\end{array}\right.
\end{aligned}
$$




$$
\begin{gathered}
\left\{\begin{array}{l}
D_{e_{1}, e_{5}}=4 \alpha_{1} D_{4}+2 \alpha_{1} D_{10}, \\
D_{e_{2}, e_{6}}=2 \alpha_{2} D_{4}+4 \alpha_{2} D_{10},
\end{array}\right. \\
\left\{\begin{array}{l}
D_{e_{1}, e_{6}}=4 \alpha_{1} D_{5}-2 \alpha_{2} D_{9}, \\
D_{e_{2}, e_{5}}=-2 \alpha_{1} D_{5}+4 \alpha_{2} D_{9},
\end{array}\right. \\
\left\{\begin{array}{l}
D_{e_{1}, e_{7}}=4 \alpha_{1} D_{6}+2 \alpha_{1} \alpha_{2} D_{8}, \\
D_{e_{2}, e_{4}}=2 D_{6}+4 \alpha_{2} D_{8},
\end{array}\right. \\
\left\{\begin{array}{l}
D_{e_{2}, e_{3}}=4 \alpha_{2} D_{7}-2 \alpha_{2} D_{12}, \\
D_{e_{4}, e_{5}}=-2 \alpha_{4} D_{7}+4 \alpha_{4} D_{12} .
\end{array}\right.
\end{gathered}
$$

In the first pair of equations, if $D_{e_{1}, e_{3}}$ and $D_{e_{4}, e_{7}}$ were dependent then $\left(4 \alpha_{1}\right)\left(4 \alpha_{4}\right)=2\left(2 \alpha_{1} \alpha_{4}\right)$. Since $12 \neq 0$, this is a contradiction. Hence the fourteen $D_{e_{i}, e_{j}}$ above are linearly independent and form a basis for $\mathfrak{D}(\mathfrak{C})$.

Theorem $1 .^{2}$ Let (5 be a Cayley algebra over a field $\Phi$ whose characteristic is not two or three. Then all derivations of $\mathbb{5}$ over $\Phi$ are inner, and have the form $\sum_{i} D_{x_{i}, y_{i}}$, for $x_{i}, y_{i}$ in (5.

In the case of characteristic three, the same sort of calculation as above will show that the $\sum D_{x_{i}, y_{i}}$ span only seven dimensions. For characteristic two, the $D_{x, y}$ need not be derivations.

\section{Whitehead's first lemma.}

THEOREM 2. Let $\mathfrak{A}$ be a separable alternative algebra over a base field $\Phi$ whose characteristic is not two or three. Let $(R, L)$ be an alternative representation of $\mathfrak{A}$ acting in the bimodule $\mathfrak{M}$. Let $f$ be a one-cocycle of $\mathfrak{A}$ into $\mathfrak{M}$, i.e., a linear transformation of $\mathfrak{A}$ into $\mathfrak{M}$ satisfying $f(a b)$ $=f(a) b+a f(b)=f(a) R_{b}+f(b) L_{a}$, for all $a, b$ in $\mathfrak{A}$. Then there exists an element $g$ belonging to the nucleus of $\mathfrak{M}$, and elements $x_{i}$ in $\mathfrak{A}, z_{i}$ in $\mathfrak{M}$, such that

$$
f(a)=[a, g]+a \sum_{i} D_{x_{i}, z_{i}}
$$

where $D_{x, z}=\left[R_{x}, R_{z}\right]+\left[R_{x}, L_{z}\right]+\left[L_{x}, L_{z}\right]$, is an inner derivation of $\mathfrak{B}=\mathfrak{A} \oplus \mathfrak{M}$, the semi-direct sum of $\mathfrak{A}$ and $\mathfrak{M}$. If the characteristic of $\Phi$ is zero, then $g$ may be taken as zero.

Proof. The proof is divided into four parts, and makes use of some of the results in [4].

${ }^{2}$ The referee has pointed out that since the $\sum_{i} D_{x_{i}, y_{i}}$ form an ideal in $\mathfrak{D}(\mathfrak{S})$, Theorem 1 is a corollary of the theorem that $\mathfrak{D}(\mathfrak{C})$ is simple. The latter result has been announced by Kaplansky in Bull. Amer. Math. Soc. vol. 60 (1954) p. 471. As far as the author knows, no proof of this result has appeared in the literature. 
(1). First we may assume that $\mathfrak{M}$ is irreducible. By [4], $\mathfrak{M}$ is completely reducible. Let $\mathfrak{M}=\sum \oplus \mathfrak{M}_{i}, \mathfrak{M}_{i}$ invariant irreducible subspaces. If $p_{i}$ is the projection of $\mathfrak{M}$ on $\mathfrak{M}_{i}$, then $f=\sum_{i} f p_{i}: f p_{i}$ is a onecocycle of $\mathfrak{A}$ into $\mathfrak{M}_{i}$. Since the nucleus of $\mathfrak{M}$ is the sum of the nuclei of the $\mathfrak{M}_{i}$, the result follows by addition.

(2). Next we show that $\mathfrak{A}$ may be assumed to be simple. Let $\mathfrak{A}=\mathfrak{A}_{1} \oplus \mathfrak{A}_{2} \oplus \cdots \oplus \mathfrak{A}_{r}, \mathfrak{A}_{i}$ simple with identity $e_{i}$. Let $\mathfrak{M}$ $=\sum_{i, j=0}^{r} \oplus \mathfrak{M}_{i j}$ be the Peirce decomposition of $\mathfrak{M}$ with respect to the $\left\{e_{i}\right\}$. The $\mathfrak{M}_{i j}$ are submodules, so by the above result, $\mathfrak{M}=\mathfrak{M}_{i j}$.

If $i=j=0$, there is nothing to prove. If $i=0$, then $\mathfrak{A}_{k}^{2}=\mathfrak{A}_{k}$ implies $f\left(\mathfrak{A}_{k}\right)=0$ for $k \neq j$. The restriction of $f$ to $\mathfrak{A}_{j}$ is a one-cocycle of $\mathfrak{A}_{j}$ into $\mathfrak{M}$. $D_{x_{j}, z}$ and $R_{\boldsymbol{o}}-L_{\boldsymbol{g}}$ are zero when acting on $\mathfrak{U}_{k}$ for $k \neq j, x_{j} \in \mathfrak{A}_{j}, z$, $g \in \mathfrak{M}=\mathfrak{M}_{0 j}$, so we may assume $\mathfrak{A}=\mathfrak{U}_{j}$. Similarly if $j=0$, we may take $\mathfrak{A}=\mathfrak{H}_{i}$.

If $\mathfrak{M}=\mathfrak{M}_{i i}$ for $i \neq 0$, then $\mathfrak{U}_{k}^{2}=\mathfrak{A}_{k}$ implies $f\left(\mathfrak{A}_{k}\right)=0$ for $k \neq i$. Since $D_{x_{i}, z}$ and $R_{\mathfrak{g}}-L_{\boldsymbol{o}}$ are zero on $\mathfrak{A}_{k}$ for $k \neq i, x_{i} \in \mathfrak{A}_{i}, z, g \in \mathfrak{M}=\mathfrak{M}_{i i}$, we may assume $\mathfrak{A}=\mathfrak{A}_{i}$.

Finally, there remains the case $\mathfrak{M}=\mathfrak{M}_{i j}, i \neq j, i \neq 0, j \neq 0$. This is an associative bimodule for $\mathfrak{A}$. In this case, $D_{x, z}$ and $R_{g}-L_{0}$ are zero on $\mathfrak{A}_{k}$ for $k \neq i, j, x \in \mathfrak{A}_{i} \oplus \mathfrak{A}_{j}, z, g \in \mathfrak{M}=\mathfrak{M}_{i j}$. So we may assume $\mathfrak{A}=\mathfrak{A}_{i} \oplus \mathfrak{A}_{j}$. But since $\mathfrak{M}_{i j}$ is associative, $\mathfrak{A}_{i}$ and $\mathfrak{A}_{j}$ cannot be Cayley algebras which are simple but nonassociative. Hence $\mathfrak{A}=\mathfrak{A}_{i} \oplus \mathfrak{A}_{j}$ is associative, and the result is well-known if we set $x_{i}=z_{i}=0$ in (3), (see [1]). Hence we may assume $\mathfrak{A}$ simple, and $\mathfrak{M}=\mathfrak{M}_{11}, \mathfrak{M}_{10}$ or $\mathfrak{M}_{01}$ in the Peirce decomposition relative to the identity of $\mathfrak{A}$.

(3). The case $\mathfrak{M}=\mathfrak{M}_{01}$ (or $\mathfrak{M}_{10}$ ).

It is easily seen $[7 ; 8]$, that the associator $(1, a, m)=0$ for $a \in \mathfrak{A}$, $m \in \mathfrak{M}$. Hence if $\mathfrak{M}=\mathfrak{M}_{01}$, then

$$
\begin{aligned}
(a, b, m) & =(a b) m-a(b m) \\
& =((a b) 1) m-a((b 1) m) \\
& =(a b)(1 m)-a(b(1 m))=0 .
\end{aligned}
$$

Hence $\mathfrak{M}$ is associative, $\mathfrak{A}$ must be associative, and the result is known by Hochschild's theorem. Similarly for $\mathfrak{M}=\mathfrak{M}_{10}$.

(4). The remaining case is $\mathfrak{M}$ a unital irreducible bimodule for the simple alternative algebra $\mathfrak{A}$. We extend the base field $\Phi$ to $\Gamma$ so that $\mathfrak{A}_{\Gamma}$ is a split algebra, i.e., either $\Gamma_{n}$, the algebra of $n \times n$ matrices over $\Gamma$, or a Cayley algebra over $\Gamma$. Also extend $\mathfrak{M}$ to $\mathfrak{M}_{\Gamma}$, an $\mathfrak{A}_{\Gamma}$-bimodule.

(a) If $\mathfrak{A}_{\Gamma}=\Gamma_{n}$, for $n \geqq 3$, then the bimodule $\mathfrak{M}_{\Gamma}$ is associative, [4]. Then $\mathfrak{M}$ is associative for $\mathfrak{A}$, and the result is known.

(b) If $\mathfrak{A}_{\Gamma}$ is a Cayley algebra over $\Gamma$, then it is easily seen, [2], that $\mathfrak{A}$ is a Cayley algebra over $\Phi$. Since the only unital irreducible 
alternative bimodule for $\mathfrak{A}=\mathbb{E}$ is $\mathfrak{E}$ itself, [4], $f$ is a derivation in $\mathfrak{E}$. The result then follows from Theorem 1, taking $g=0$.

(c) If $\mathfrak{A}_{\Gamma}=\Gamma_{2}$, and $\mathfrak{M}_{\Gamma}$ is an associative $\Gamma_{2}$-bimodule, then $\mathfrak{M}$ is an associative $\mathfrak{A}$-bimodule, and the result follows as before. By [4], the only other possibility is that $\mathfrak{A}_{\Gamma}=\Gamma_{2}$, and $\mathfrak{M}_{\Gamma}$ is a Cayley bimodule for $\mathfrak{A}_{\Gamma}$. In this case, the mappings $a \rightarrow R_{a}, a \rightarrow L_{a}$ are respectively an anti-homomorphism and a homomorphism of $\mathfrak{A}$ into the algebra of linear transformations of $\mathfrak{M}$. Thus $\mathfrak{M}$ is a Cayley bimodule for $\mathfrak{A}$. We now give a separate calculation for this last case.

Let $e_{11}, e_{12}, e_{21}, e_{22}$ be the matrix units of $\Phi_{2}, \mathfrak{M}$ the vector space of two dimensions acted on by $\Phi_{2}$. $\mathfrak{M}$ has a basis $v_{1}=(1,0), v_{2}=(0,1)$. Let $a \rightarrow U_{a}$ be the representation of $\Phi_{2}$ on $\mathfrak{M}$. Then $\mathfrak{M}$ is a Cayley bimodule for $\Phi_{2}$ under $\left(R_{a}, L_{a}\right)$, where $R_{a}=U_{\bar{a}}, L_{a}=U_{a}$, where $\tilde{a}$ $=($ trace $a) 1-a$ is an involution in $\Phi_{2} . \mathfrak{M}$ is a unital alternative $\Phi_{2^{-}}$ bimodule, and $a \rightarrow L_{a}, a \rightarrow R_{a}$ are a homomorphism and an antihomomorphism respectively. Let $\mathfrak{D}$ be the vector space of all derivations (one-cocycles) of $\Phi_{2}$ into $\mathfrak{M}$. Any element of $\mathfrak{D}$ is uniquely determined by its effect on the generators $e_{12}, e_{21}$ of $\Phi_{2}$, and hence $\mathfrak{D}$ is fourdimensional over $\Phi$. We exhibit a basis $D_{1}, D_{2}, D_{3}, D_{4}$ as follows:

\begin{tabular}{c|c|c|} 
& $e_{12}$ & $e_{21}$ \\
\hline$D_{1}$ & $v_{1}$ & 0 \\
\hline$D_{2}$ & $v_{2}$ & 0 \\
\hline$D_{3}$ & 0 & $v_{1}$ \\
\hline$D_{4}$ & 0 & $v_{2}$
\end{tabular}

If $a \in \Phi_{2}, v \in \mathfrak{M}$, then $D_{a, v}=\left[R_{a}, R_{v}\right]+\left[R_{a}, L_{v}\right]+\left[L_{a}, L_{v}\right]$ is a derivation of the semi-direct sum $\Phi_{2} \oplus \mathfrak{M}$, mapping $\Phi_{2}$ into $\mathfrak{M}$, and $\mathfrak{M}$ into $\{0\}$. Its restriction to $\Phi_{2}$ may be considered as an element of $\mathfrak{D}$. We show that these $D_{a, v}$ span $\mathfrak{D}$, in which case we have the desired form (1) for $f \in \mathfrak{D}$, with $g=0$. The calculation is similar to the one in $\S 2$.

\begin{tabular}{c|c|c|} 
& $e_{12}$ & $e_{21}$ \\
\hline$D_{e_{12}, v_{1}}$ & 0 & $v_{1}$ \\
\hline$D_{e_{12}, v_{2}}$ & 0 & $3 v_{2}$ \\
\hline$D_{e_{21}, v_{1}}$ & $3 v_{1}$ & 0 \\
\hline$D_{e_{11}, v_{2}}$ & $v_{2}$ & 0
\end{tabular}

$$
\begin{aligned}
& D_{e_{12, v_{1}}}=D_{3} \\
& D_{e_{12, v_{2}}}=3 D_{4} \\
& D_{e_{21}, v_{1}}=3 D_{1} \\
& D_{e_{21}, v_{2}}=D_{2}
\end{aligned}
$$

This completes the proof, except for the last statement. 
If characteristic $\Phi=0$, then to show $g=0$, it again suffices to take $\mathfrak{M}$ irreducible, $\mathfrak{A}$ simple. If $\mathfrak{A}$ is a Cayley algebra or if $\mathfrak{A}$ is $\Phi_{2}, \mathfrak{M}$ a Cayley bimodule, then we have seen that $g=0$. If $\mathfrak{A}=\Phi_{n}, n \geqq 2, \mathfrak{M}$ associative, then we may take $\mathfrak{M}=\Phi_{n}$, and the representation as the biregular one. Then $f(a)=a\left(R_{g}-L_{g}\right)$, for $g \in \Phi_{n}$. However, $\mathfrak{A}=\mathfrak{c} \oplus \mathfrak{A}^{1}$, $\sqrt{5}$ the center of $\mathfrak{A}, \mathfrak{A}^{1}$ the space spanned by the commutators $[a, b]$ $=a b-b a$ in $\mathfrak{A}$. Let $g=c+\sum_{i}\left[a_{i}, b_{i}\right]$. Then $R_{g}-L_{g}=\sum_{i} D_{a_{i}, b_{i}}$, and this proves the last statement of the theorem.

CoROllary. If $\mathfrak{A}$ is a separable subalgebra of an alternative algebra $\mathfrak{B}$ of characteristic not two or three, then any derivation of $\mathfrak{A}$ into $\mathfrak{B}$ can be extended to an inner derivation of $\mathfrak{B}$ which has the form $R_{\sigma}-L_{g}$ $+\sum D_{x_{i}, y_{i}}$, for $g$ in the nucleus of $\mathfrak{B}, x_{i}, y_{i} \in \mathfrak{B}$. If characteristic $\Phi=0$, then $g$ may be taken as zero.

REMARKS. We remark that for characteristic zero, our theorem is Theorems 3 and 4 of [6].

One can use Theorem 2 to show that if $\mathfrak{A}$ is an alternative algebra of characteristic not two or three, $\Re$ its radical, $\mathfrak{A} / \mathfrak{N}$ separable, and $\mathfrak{\Im}_{1}, \mathfrak{S}_{2}$ two separable subalgebras of $\mathfrak{A}$ isomorphic with $\mathfrak{A} / \mathfrak{R}$, then there exists a nonsingular linear transformation $G=I+D, D$ in the radical of the enveloping associative algebra of $\mathfrak{A}$, such that $\mathfrak{S}_{1}^{G}=\mathfrak{S}_{2}$.

Finally we note that our examples showed that Theorem 2 is false for characteristic three. In this case, commutation by any element of $\mathfrak{M}$ is a one-cocycle. However, even if one removes the restriction that $g$ be in the nucleus of $\mathfrak{M}$, the Cayley algebra will still provide a counter-example to Theorem 2 for characteristic three.

\section{REFERENCES}

1. G. Hochschild, The cohomology groups of an associative algebra, Ann. of Math (2) vol. 46 (1945).

2. N. Jacobson, Cayley numbers and normal simple Lie algebras, Duke Math. J. vol. 5 (1939).

3. - General representation theory of Jordan algebras, Trans. Amer. Math. Soc. vol. 70 (1951).

4. - Structure of alternative and Jordan bimodules, Osaka Math. J. vol. 6 (1954).

5. R. D. Schafer, Inner derivations of nonassociative algebras, Bull. Amer. Math. Soc. vol. 55 (1949).

6. - Representations of alternative algebras, Trans. Amer. Math. Soc. vol. 72 (1952).

7. M. Zorn, Theorie der alternativen Ringe, Abh. Math. Sem. Hamburgischen Univ. vol. 8 (1930).

8. - Alternative rings and related questions I: Existence of the radical, Ann. of Math. (2) vol. 42 (1941).

YALE UNIVERSITY 